\title{
Ralph Miliband Der kapitalistische Staat: Antwort an Nicos Poulantzas
}

\begin{abstract}
$[\ldots]$
Während ein Teil der Kritik von Poulantzas - wie ich zu zeigen versuchen werde - unberechtigt ist, ist das Ziel meiner folgenden Bemerkungen nur beiläufig, mein Buch zu »verteidigen «. Mein Hauptziel ist eher, einige generelle Gesichtspunkte aufzugreifen, die sich aus seiner Rezension ergeben und die mir von besonderem Interesse für die Untersuchung von Natur und Rolle des Staates in der kapitalistischen Gesellschaft zu sein scheinen. [...]
\end{abstract}

\section{Das Problem der Methode}

Der erste dieser Gesichtspunkre betrifft die Frage der Methode. Poulantzas deuter an, da $B$, ungeachter der Verdienste des Buches (über die er sich sehr großzügig äußert), die in ihm versuchte Analyse durch das Fehlen eines »Problemzusammenhanges « beeinträchtigt wird, in den die aufgewiesenen konkreten $\mathrm{Da}$ ten sich einfügten. Letztlich wirfe mir P. das vor, was C. Wright Mills *abstrakten Empirismusa nannte und was ich selbst gerade pluralistischen Autoren vorwerfe. ${ }^{1}$

Poulantzas stellt ganz richtig fest, daß es eine Vorbedingung jeder wissenschaftlichen Untersuchung des *Konkreten « ist, die erkenntnistheoretischen Grundlagen der eigenen Auseinandersetzung mit ihm offenzulegen, und er fährt fort mit der Bemerkung, daß $»$ Miliband an keiner Stelle die marxistische Staztstheorie als solche behandelt, obgleich sie ständig in seinem Werk implizit enthalten ist". Tatsächlich gebe ich ganz explizit einen Uberblick über die marxistische Theoric des Staates², zweifellos aber sehr kurz. Ein Grund hierfür ist - abgesehen davon, $\mathrm{da}$ ich an an anderer Stelle die Marx'sche Theorie des Staates erörtert ha$b^{3}{ }^{3}$, daB ich mich, nachdem ich die marxistische Theorie des Staates skizziert hatte, darauf konzentrierte, sie der herrschenden demokratisch-pluralistischen Position entgegenzusetzen und deren Unzulänglichkeiten in der mir einzig möglich erscheinenden Form, nämlich mit empirischen Mitteln, zu zeigen. Es ist vollkommen richtig, wenn Poulantzas die Wichtigkeit eines angemessenen Problemzusammenhanges für ein solches Unternehmen betont; und es ist wahrscheinlich richtig, daß mein Problemzusammenhang ungenügend erklärt ist. Aber da Poulantzas feststellt, daß ein derartiger Problemzusammenhang »ständig in meinem Werk implizit enthalten ist $\alpha$, bezweifle ich, daß meine Darstellung durch empiristische Deformationen derart beeinträchtigt wird, wie er meint. Das heißt, daß

\footnotetext{
1 The Seare in Capicalise Socicty, S. 172.

- Ebenda, S. 5, 93.

3 Marx and the State, in: The Socialist Register, 1965.
} 
der geforderte Problemzusammenhang in meiner Arbeit nicht fehlt, und daß ich nicht deswegen dazu veranlaßt werde, *die bürgerlichen Ideologien vom Staat anzugreifen und (mich) dabei selbst auf deren eigenen Boden zu begeben*.

Poulantzas führt als Beispicl für diesen angeblichen Mangel die Tarsache an, daß ich [...] es fälschlicherweise unterlasse, eine Kritik der ideologischen Karegorie Elite zu liefern und mich deshalb innerhalb des Problemzusammenhanges bewege, den ich zu bekämpfen versuche. Auch hier bezweifle ich jedoch, daß die Anmerkung berechtigt ist. Ich bin mir bewußt, bis zu welchem Grad bestimmte Worte und Konzeptionen ideologisch und politisch aufgeladen sind, und habe in der Tar eine Anzahl von Beispielen angeführt, die von einem »unschuldigen * Gebrauch weit entfernt sind. ${ }^{4}$ Gerade aus diesem Grund zögerte ich, von »Eliten a zu sprechen. Schlicßlich habe ich mich doch dazu entschlossen, einmal, weil ich annahm - vielleicht fälschlicherweise -, daß dieser Begriff mittlerweile einen genügend neutralen Inhait angenommen habe (nebenbei gesagt mag er im Französischen eine sehr viel ideologischere Tönung haben als im Englischen), zum anderen, weil er, in seinem neurralen Sinn, als das brauchbarste Wort erschien, um den Grundtatbestand auszudrücken, daß, während tatsächlich innerhalb der herrschenden Klasse solche getrennte „Eliten « bestehen, die Poulantzas mit dem zugegebenermaßen neutraleren, aber ziemlich schwachen Wort »Fraktionen * beschreibt, diese vollständig mit der Existenz einer herrschenden Klasse vereinbar und in der Tat Teile dieser Klasse sind. Poulantzas behaupter, da $B$ die »konkrete Wirklichkeit«, die durch die Vorsteliung eines »Pluralismus von Eliten * verschleiert wird, nur zu erfassen ist, "wenn genau die Kategorie der Elite zurückgewiesen wird . Ich würde selbst sagen, daß die konkrete Realität nur zu erfassen ist, wenn das Konzept der Elite gegen diejenigen gewandt, die es für apologetische $Z$ wecke verwenden, und an $\mathrm{ihm}$ aufgewiesen wird, daB es notwendig in das Konzepr einer herrschenden Klasse gehört. D. h. es gibt Konzepte der bürgerlichen Sozialwissenschaft, die für kritische ebensogut wie für apologetische Zwecke verwendet werden können. Der Versuch mag oft riskant sein, ist aber zuweilen legitim und notwendig.

Die Grundfrage jedoch, die Poulantzas aufwirft, geht weit über die Verwendung dicses oder jenes Konzepts hinaus. Sie betrifft in der Tat nichts Geringeres als den Status der empirischen Forschung und ihre Beziehung zur Theorie. In dieser Hinsicht würde ich sofort zugestehen, daß • The State in Capitalist Society * zuwenig *theoretisch « scin mag in dem Sinne, wie Poulantzas es versteht; aber ich neige auch der Auffassung zu, daß sein eigener Ansatz, so wie er in seiner Rezension und seinem im übrigen wichtigen Buch »Pouvoir Politique et Classes Sociales d dargcstellt ist, in entgegengesetzter Richtung fehlgeht. Um es klar zu sagen, ich glaube, man kann - wenigstens auf diesem Gebiet - so sehr damit beschäftigt sein, einen angemessenen Problemzusammenhang auszuarbeiten und dabei jede Entstellung durch den gegnerischen zu vermeiden, daß man darüber den Blick für die unbedingte Notwendigkeit empirischer Forschung und der empirischen Falsifikation jener gegnerischen apologetischen Problemzusammenhänge verliert. Poulantzas erklärt, daß er sich nicht gegen das Studium des -Konkreten« wende. Ich würde schr viel weiter gehen und sagen, daß solch ein

1 Z. B.: $\rightarrow$ Die Regierungen mögen einzig mit dem besseren Fortschreiten der $\rightarrow$ Wirtschaft * beschäftigt sein. Aber die Beschreibung des Systems als $₫$ die Wirtschafe $\propto$ ist Teil des ideologischen Jargons und verdunkelt den realen Prozeß. Denn was verbessert wird, ist die kaptalistusche Wirtschaft und dies gewälnleister, daß - wer immer dabei profitieren oder niche profitieren mag - kapitalistische Interessen die letzren sein werden, die zu kurz kommen.e (A. з. O., S. 79. Gesperrt im Texr). 
Studium des Konkreten - natürlich auf der Basis eines angemessenen Problemzusammenhanges - die conditio sine qua non dieser Art von Entschleierungsvorhaben ist, wie es - erklärt er freundlicherweise - meinem Buch gelingt. Es war schließlich kein anderer als Marx, der die Bedeutung der empirischen Verifikation (oder Falsifikation) hervorhob und der viele Jahre seines Iebens gerade mit einem derartigen Unternehmen beschäftigt war. Und obgleich ich niemals behaupte, daß Poulantzas sich dieser Tatsache nicht bewußt wäre, glaube ich doch, daß er dazu neigr - dies gilt auch für Louis Althusser und scine Mitarbeiter -, ihr weniger Aufmerksamkeit zuzuwenden, als sie verdient. Ich muß betonen, dies ist keine krude (und falsche) Gegenposition empirischer gegenüber nicht- oder antiempirischer Ansätzen: Es ist eine Frage der Betonung, aber die Betonung ist wichtig.

\section{Die objektive Natur des Staates}

Poulantzas' Kritik meines Ansatzes bringt weitere Differenzen zwischen uns zum Ausdruck. Ehe ich hierauf eingche, möchre ich ganz kurz das aufgreifen, was er "das Scheinproblem der Managerherrschaft « nennt. Die Managerherrschaft ist ein Scheinproblem in einem Sinne, in einem anderen nicht. Sie ist ein Scheinproblem in dem Sinne, daß die "Motivationen « der Manager (über die ich gleich sprechen werde) nicht so gestaltet sind, $\mathrm{daB}$ man sie in irgendeiner grundlegenden Weise von anderen Mitgliedern der kapitalistischen Klasse unterscheiden könnte. D. h. wir stimmen darin überein, daß die These vom »Unternehmen mit Herz " eine Verschleierung darstellt. Aber er meint auch, daß ich den Managern meine Bedeutung beimesse, die sie nicht besitzen«. Damit wird scheint mir - die Bedeutung des Phänomens der Manager in der inneren Organisation der kapitalistischen Produktion unterstützt - was, nebenbei gesagt, Marx, der vor hundert Jahren schrieb, nicht tat. ${ }^{5}$ Poulantzas selbst hebr mehr die "Differenzen und Beziehungen zwischen den Fraktionen des Kapitals» hervor. Diese sind zwar wichrig und müssen in eine ökonomische und politische Analyse des gegenwärtigen Kapitalismus einbezogen sein; aber ich se!bst würde darauf bestehen, daß die Betonung, die er auf die Differenzen und Beziehungen legt, leicht den zugruride liegenden Zusammenhalt dieser verschiedenen Elemente verdunkeln und geradezu denen in die Hände spielen kann, die diese Differenzen in den Mittelpunkt stellen, um den fundamentalen Zusammenhalt der kapitalistischen Klasse unter den Bedingungen des fortgeschrittenen Kapitalismus zu leugnen.

Wichtiger ist jedoch, daß Poulantzas erklärt, ich messe den »Motivationen « der Manager übermäßige Bedeutung bei, ja, ich sei völlig im Irrtum darin, ihnen überhaupt Bedeutung beizumessen. [...]

Ignoriert man sie überhaupt, so läßt man eine gefährliche Lücke in der Beweis-

* Anm. d. Obers.: engl. soulful corporation 4 . Gemeint ist die These, derzufolge unter der Managerherrschaft nicht mehr die Profitorientierung, sondern die Wahrnehmung des Allgemenninteresses die Unternehmenspolitik bestimme. Darstellung und Kritik der These vom - Unternehmen mis Herz a bei Baran/Sweezy, Monopolkapital, S. zo.

5 Tatsächlich gehen scine Formulierungen vielleicht weiter, als es gerechtfertigr ist: \$... ein großer Teil des gesellschaftlichen Kapitals (wird) von den Nichtesgentümern desselben $\pm n z e-$ wandt, die gınz anders ins Zeug gehen als der ... Eigentümer, .... (Das Kapital Bd. III, MEW Bd. 25, Berlin 1966, S. 457. Es ist die Aufhebung der kapitalistischen Produkcions: weise innerhalb der kapitalistischen Produktionsweise selbst, und daher ein sich selbst aufhebender Widerspruch, der prima facie als bloßser Ubergangspunkt zu einer neuen Produktıonsform sıch darstellt.\& A. 2. O., S. 454. 
führung, die gegen die Manager-Apologetik ins Feld geführt werden muß. Aus diesem Grund, meine ich, widmen beispielsweise Baran und Sweezy in ihrem - Monopolkapital d der Unternehmenspolitik beträchtliche Aufmerksamkeit. Diese Streitfrage der "Motivationen « taucht auch - in einer sehr viel signifikanteren und weiterreichenden Weise - im Zusammenhang mit dem auf, was ich die staatliche Elite und ihr Verhältnis zur herrschenden Klasse nannte. Poulantzas bemerkt, daß ich - um die Ideologien, die ciner Neutralität des Staates das Wort reden, zu widerlegen - den Beweis dafür antrete, daß Mitglieder dieser Klasse selbst mit der Regierung verzahnt sind, und weiter zeige, in welchem Grade die Inhaber der Schlüsselpositionen der verschiedenen Teile des Staatsapparates durch soziale Herkunft, Status, Milieu (und, hätte er hinzufügen können, ideologische Disposition) der herrschenden Klasse verbunden sind. Aber, fügt er hinzu, dieses Verfahren sei, wenn ihm auch "zentrale entscbleiernde Bedeutung ${ }^{\beta}$ zulcomme, »nicht das bedeutsamste*. Seine Begründung dafür ist so grundsätzlich, daß ich sie hier ausführlich zitieren muß: „Das Verhältnis $z$ wischen der bürgerlichen Klasse und dem Staat ist ein objekttves. Das heißr: Wenn die Funktion des States in einer bestimmten sozialen Formation sich mit den Interessen der herrschenden Klasse in dieser Formation deckt, so geschieht dies auf Grund des Systems selbst. $\alpha^{\top}$ So fungieren die Mitglieder des Staatsapparates auch wentsprechend einem spezifischen Systemzusammenhang. Ihre klassenmäßige Herkunft tritt hinter dem zurück, was sie zusammenhält - ihrer Klassenfunktion: also der Tatsache, daß sie eben zum Staatsapparat gehören und als ihre objektive Funktion die Aktualisierung der Rolle des Staates vorfinden. Die Totalität dieser Rolle deckt sich mit den Interessen der herrschenden Klasse*. ${ }^{8}$

Ich möchte hierzu zwei Bemerkungen machen. Die erste und weniger wichtige ist die, daß Poulantzas stark das Ausmaß unterschätze, in dem ich selbst den -objektiven Verhältnissen «, die die Rolle des Staates beeinflussen und formen, Rechnung trage. Tatsächlich stelle ich wiederholt fest, wie Regierung und Bürokratie, unabhängig von ihrer Klassenherkunft und sogar ihrer ideologischen Disposition, den strukturellen Zwängen des Systems unterworfen sind. Trotzdem hätte ich vielleicht diesen Aspekt des Problems mehr hervorheben sollen. Unabhängig davon aber meine ich - und dies ist mein zweiter Gesichtspunkt -, $\mathrm{da} B$ Poulantzas selbst hier ziemlich einseitig verfährt und viel zu weit geht, wenn er die Eigenart der staatlichen Elite als völlig bedeutungslos abtut. Seine ausschließliche Betonung der »objelstiven Verhältnisse « legt nämlich die Annahme nahe, was der Staat tue, sei in aller Einzelheit und zu allen Zeiten abschließend determiniert durch diese objektiven Verhältnisse: mit anderen Worten, die strukturellen Zwänge des Systems wirken so absolut, daß sie die, die den Staat in Gang halten, zu bloßen Funktionären und Vollstreckern einer Politik machen, die ihnen durch »das System * aufgezwungen wird. Gleichzeitig verwirft er aber auch »die lange marxistische Tradition, die im Staat ein einfaches - durch die herrschende Klasse nach Belieben zu manipulierendes - Werkzeug oder Instrument sah«. Stattdessen hebt er die »relative Autonomie des Staates* hervor. Damit ist meines Erachtens aber nur erreicht, daß die Begriffe der »objektiven Strukturen « und der »objektiven Verhältnisse « an die Stelle des Begriffs der "herrschenden Klasse getreten sind. Da nun aber die herrschende Klasse

\footnotetext{
- Gesperrt in Text.

7 Gespert in Text.

- Gesperre im Text.
} 
ein dominierendes Element des Systems darstellt, sind wir tatsächlich wieder am Ausgangspunkt angelangt, der totalen Unterordnung der staatlichen Elite unter jene Klasse. Das heißt, der Staat wird nicht von der herrschenden Klasse dazu smanipuliert «, sich ihren Wünschen zu fügen: er tut das autonom, aber auch total, und zwar auf Grund der mobjektiven Verhältnisse «, die ihm das System aufzwingt. Poulantzas verurtcilt den ○/konomismus « der $Z_{\text {weiten }}$ und Dritten Internationale und führt deren Vernachlässigung des Staates auf ihn zurück. Aber seine eigene Analyse scheint mir geradewegs zu einer Art strukturalem Determinismus oder gar einem strukturalen Superdeterminismus zu führen, der eine realitätsgetreue Einschätzung des dialektischen Verhältnisses zwischen dem Staat und $»$ dem System $\propto$ unmöglich macht.

Ich für meinen Teil glaube, $» d a B$ der Staat in diesen Klassengesellschaften primär und unvermeidbar der Beschützer und Gönner der ökonomischen Interessen ist, die in ihnen vorherrschen. Seine swirkliche « Absicht und Aufgabe ist, deren weitere Vorherrschaft zu sichern, nicht sie zu verhindern $«,{ }^{2}[\ldots]$

Die politische Gefahr eines strukturalen Superdeterminismus erscheint mir offenkundig. Denn wenn - wie angedeutet wird - die staatliche Elite vollkommen von objektiven Strukturen gefangengehalten wird, so folgr daraus, daß $\gg$ real « kein Unterschied besteht $z$ wischen einem Staat, der z. B. von bürgerlichen Konstitutionalisten, ob konservativen oder sozialdemokratischen, und einem, der beispielsweise von Faschisten regiert wird. Genau dieselbe Einstellung verführte die Komintern in ihrer Klasse gegen Klasse-Periode « dazu, fatalerweise zu unterschätzen, was der Sieg der Nazis für die deutsche Arbeiterbewegung bedeuten würde. Dies ist eine ultra-linke $A b w c i c h u n g$, die auch heute nicht ungewöhnlich ist. Sie ist das Gegenstück zu einer rechten Abwcichung, die davon ausgeht, daß Veränderungen in der Regierung, z. B. die Wahl einer sozialdemokratischen Regierung, verbunden mit einigen Änderungen im Personal des Staatsapparates, genügten, um der Natur und Rolle des Staates einen vollkommen neuen Charak- ter zu verleihen. Beides sind Abweichungen, und beide sind gefährlich.

Die gleiche Art, Unterschiede in den Formen der Regierung und des Staates auszulöschen, kommt bei Poulantzas' Bemerkungen über die »relative Autonomiex des Staates zum Vorschein. Er behaupter, Marx habe den Bonapartismus als "Religion der Boụrgeoisie " gekennzeichnet, und unterstellt Marx die Ansicht, der Bonapartismus sei für »alle Formen des kapitalistischen Staates charakteristisch 4.10 Vielleicht werde ich eines Besseren belehrt werden: aber ich kenne keine Schrift von Marx, die eine solche Interpretation zuläßt. Hätte er tatsächlich etwas geschrieben, das eine solche Interpretation zuließe, so hätte er sich schwer geirrt. Welche Bedeutung man diesem Konzept auch immer zuschreibt: der Bonapartismus ist nicht für alle bisherigen Formen des kapitalistischen Staztes charakteristisch, eher das Gegenteil. Tatsächlich sagte Marx, daß der Ronapartismus in Frankreich "die einzig mögliche Regierungsform (war), zu einer Zeit, wo die Bourgeoisie die Fähigkeit, die Nation zu beherrschen, schon verloren und wo die Arbeiterklasse diese Fähigkeit noch nicht erworben hatte ${ }^{11}$ Es ist vollkommen richtig, daß alle Staaten in gewissem Ausmaß "autonom " sind. Poulantzas mißversteht mich, wenn er behauptet, daß ich »diese Autonomie schließlich nur für den Extremfall des Faschismus zugestehe«.

9. A. O., S. 265 .

10 Gesperrt in Text.

in Der Bürgcrkrieg in Frankreich, in: Politische Sehriften, ed. Lieber Scuttgart 1960, Bd. II. S. $92 \mathrm{r}$. 
Tatsächlich sage ich, daß der Faschismus der Extremfall der Autonomie des staates im Kontext einer kapitalistischen Geseilschaft ist - was keineswegs dasselbe ist - und daß zwischen der Art von Autonomie, die der Staat uncer dem Faschismus erlangt, und der, die er unter den Bedingungen bürgerlicher Demokratie erlangt, eine breite Kluft liegt, die zu unterschätzen gefährlich ist. Das veranlaßr mich niche zu einer Verherrlichung bürgerlicher Demokratie. Es veranlaßr mich viclmehr zu sagen, „daß der Gegenstand der sozialistischen Kririk „bürgerlicher Freiheiten « niche ist (oder sein sollte), daß sie bedeutungslos seien, sondern, daß sie im Kern unzulänglich sind und durch die radikale Veränderung des ökonomischen, politischen und sozialen Kontexts, der sie zu Unzulänglichkeit und Aushöhlung verdammr, ausgeweitet werden müssen «. ${ }^{12}$

\section{Die ideologischen Institutionen}

$[\ldots]$

Was meine Analyse wert sein mag, liegr wohl darin, daß ich den Tatbestand zu demonstrieren versucht habe, daß die "politische Sozialisation « ein Prozeß ist, der von Institutionen betrieben wird, von denen viele nicht müde werden, auf ihrem »unideologischen«, »unpolitischen« und »neutralen« Charakter zu insistieren.

Viel wichtiger ist, daß Poulantzas erklärt, diese Institutionen ngehören zum System des Staates" und die These aufstellt, daß dieses System des Staates maus mebreren Apparaten oder Institutionen (bestehr), von denen manche eine überwiegend repressive, andere eine überwiegend ideologische Funkrion ausüben «. Zu den letzreren zählt er die Kirche, politische Parteien, Gewerkschaften, Schulen, Massenmedien und, von einer bestimmten Warte aus, die Familie. ${ }^{13}$

Demgegenüber habe ich allergrößte Vorbehalte. Ich erkläre in »The Stare in Capitalist Society «, daß der Staat in steigendem Maße in den Prozeß der »politischen Sozialisation « einbezogen ist und $d a ß$ er in gewisser Hinsicht dabei eine entscheidende Rolle spieit. ${ }^{14}$ Genauso wie es notwendig ist zu zeigen, daß die genannten Institutionen Teile eines Herrschaftssystems sind und daß sie, wie Poulantzas erklärt, zunehmend an den Staar gekettet und von ihm gestützr werden, ebenso wichtig ist es meines Erachtens aber auch, nicht die Tatsache zu verwischen, daß sie in bürgerlichen Demokratien nicht Teil des Staates, sondern des politischen Systems sind. Die Institutionen unterliegen in steigendem Maße einem Prozeß der "Verstaatlichung". Wie ich in meinem Buch feststelle, wird dieser Prozeß sicherlich noch durch die Tatsache verstärkt werden, daß der Staat unter den Bedingungen der permanenten Krise des fortgeschrittenen $\mathrm{Ka}$ pitalismus immer umfassendere Zuständigkeit für politische Indoktrination und Verschleierung an sich ziehen muß. Aber anzunehmen, die relevanten Institutionen seien bereits Teil des staatlichen Systems, scheint mir nicht mit der Wirklichkeir übereinzustimmen und tendiert dahin, die diesbezügliche Differenz zwischen diesen politischen Systemen und jenen, in denen die ideologischen Institutionen tatsächlich Teil eines staatsmonopolistischen Herrschaftssystems sind, zu verwischen. In den erstgenannten Systemen behalten die ideologischen Institu-

12 Ebenda, S. 267.

13 Gesperte im Texe.

14 A. a. O., S. I $83 f f$. 
tionen ein sehr weites Ausmaß an Autonomic; deshalb fällt es ihnen auch leichter zu verschleiern, in welchem Ausmaß sie zum Herrschaftssystem der kapitalistischen Gesellschaft gehören. Um zu zeigen, daß sie dazu gehören, braucht man niche etwa zu bchaupten, sie seien Teil des staatlichen Systems, sondern muß man zeigen, wie sie ihre ideologischen Funktionen außerhalb dieses Systems ausüben; und dies habe ich zu tun versuche.

$[\ldots]$ 